\title{
A New Test for Unknown Age Class of Life Distribution based on Laplace Transform Technique
}

\author{
S. E. Abu-Youssef \\ Department of Mathematics, \\ Faculty of Science, \\ Al-Azhar University, Cairo, \\ Egypt
}

\author{
Nahd S. A. Ali \\ Department of Mathematics, \\ Faculty of Education, \\ Ain Shams University, Cairo, \\ Egypt
}

\author{
M. E. Bakr \\ Egyptian General Authority for \\ Meteorology, Ministry of Civil \\ Aviation, Cairo, Egypt
}

\begin{abstract}
Most of the approaches suggested during the last decades for solving life testing problems are markedly different from those used in the related but wider area of Laplace transform technique. In this paper, it is demonstrated that applying the Laplace transform technique makes sense also for solving life testing problems and that result in simpler procedures that are asymptotically equivalent or better than standard ones. A new test statistics for testing exponentiality against used better than age in the Laplace transform order aging class of life distribution (UBAL) is proposed. Pitman's asymptotic efficiencies of this test are calculated and compared with other tests. The percentiles of this test statistic are tabulated for censored and non-censored data. Finally, examples in different areas are used as practical applications of the proposed test.
\end{abstract}

\section{Keywords}

UBA and UBAL classes of life distributions; Testing hypothesis; Right censored data; Makeham, Weibull, Linear failure rate (LFR) distributions.

\section{INTRODUCTION}

Statistical inferences are used to project the data from the sample to the entire population. Statistical inference based on two main branches one of them the estimation and the other is testing hypotheses. In general, we do not know the true value (claim) of population parameters they must be estimated. However, we do have hypotheses about what the true values (claims) are. The hypothesis actually to be tested is usually given the symbol $\mathrm{H}_{0}$, and is commonly referred to as the null hypothesis. The other hypothesis, which is assumed to be true when the null hypothesis is false, is referred to as the alternative hypothesis, and is often symbolized $\mathrm{H}_{1}$. Both the null and alternative hypothesis should be stated before any statistical test of significance is conducted.

In the last decade, various classes of life distributions have been proposed in order to model different aspects of aging. We get the well-known classes of increasing failure rate (IFR), increasing failure rate on average (IFRA), and used better than aged (UBA), used better than aged in expectation (UBAE). Properties and applications of these aging notions can be found, for instance, in Bryson and Siddiqui (1969), Barlow and Proschan (1981), Ahmad (2004).

In this paper, we interest in the used better than aged in Laplace transform order aging class of life distribution UBAL which introduced by Abu-Youssef and Bakr (2018).

A real data is given and we desire to test $\mathrm{H}_{0}$ : data is exponential versus the alternative hypothesis $\mathrm{H}_{1}$ : data is not exponential. To choose between $\mathrm{H}_{0}$ and $\mathrm{H}_{1}$ or to make a decision we need to define test statistic. The test statistic is a random variable used to determine how close a specific sample result falls to one of the hypotheses being tested.

In reliability theory, aging life is usually characterized by a nonnegative continuous random variable $X \geq 0$ representing equipment life with distribution function $F$ and survival function $\bar{F}(\mathrm{t})=1-\mathrm{F}(\mathrm{t})$ such that $\mathrm{F}(0-)=0$. One of the most important approaches to the study of aging is based on the concept of the residual life. For any random variable $\mathrm{X}$, let $\mathrm{X}_{\mathrm{t}}=[\mathrm{X}-\mathrm{t} \mid \mathrm{X}>t], \mathrm{t} \in\{\mathrm{x}: \mathrm{F}(\mathrm{x})<1)$, denote a random variable whose distribution is the same as the conditional distribution of $\mathrm{X}-\mathrm{t}$ given that $\mathrm{X}>t$ and has survival function

$\overline{\mathrm{F}}_{\mathrm{t}}(\mathrm{x})=\left\{\begin{array}{cc}\frac{\overline{\mathrm{F}}(\mathrm{x}+\mathrm{t})}{\overline{\mathrm{F}}(\mathrm{t})} & \overline{\mathrm{F}}(\mathrm{t})>0 \\ 0 & \overline{\mathrm{F}}(\mathrm{t})=0\end{array}\right.$.

When $\mathrm{X}$ is the lifetime of a device which has a finite mean $\mu=\mathrm{E}(\mathrm{X})=\int_{0}^{\infty} \overline{\mathrm{F}}(\mathrm{u}) \mathrm{du}$, the mean of $\mathrm{X}_{\mathrm{t}}$ is called mean residual life (MRL) and is given by

$\mu(t)=E\left(X_{t}\right)=\frac{\int_{t}^{\infty} \bar{F}(u) d u}{\bar{F}(t)}$.

Further, the hazard rate of $\mathrm{X}$ is defined by

$$
h(t)=-\frac{d}{d t} \ln \bar{F}(t)=\frac{f(t)}{\bar{F}(t)}, t \geq 0, \bar{F}(t)>0,
$$

where $f(t)=F^{\prime}(t)$ is the probability density of $X$ assuming it exist. Note that if $\lim _{t \rightarrow \infty} h(t)=h(\infty)$ it exists and is positive, then (Willmot and Cai (2000))

$\mu(\infty)=\lim _{t \rightarrow \infty} \mu(t)=\frac{1}{h(\infty)}$.

We review some common notions of stochastic orderings and aging notions are considered in this paper (see Barlow and Proschan (1981)).

If $\mathrm{X}$ and $\mathrm{Y}$ are two random variables with distributions $\mathrm{F}$ and $\mathrm{G}$ (survivals $\bar{F}$ and $\overline{\mathrm{G}}$ ), respectively, then we say that $\mathrm{X}$ is smaller than $\mathrm{Y}$ in the:

$$
\begin{aligned}
& \text { a) Usual stochastic order, denoted by } \\
& \mathrm{X} \leq_{\text {st }} \mathrm{Y} \text { if } \\
& \overline{\mathrm{F}}(\mathrm{x}) \leq \overline{\mathrm{G}}(\mathrm{x}) \text { for all } \mathrm{x} \text {. } \\
& \text { b) Increasing convex order, denoted by } \\
& \mathrm{X} \leq_{\mathrm{icx}} \mathrm{Y} \text { if } \\
& \int_{x}^{\infty} \bar{F}(u) d u \leq \int_{x}^{\infty} \bar{G}(u) d u . \\
& \text { c) Increasing concave order, denoted by } \\
& \mathrm{X} \leq_{\mathrm{icv}} \mathrm{Y} \text { if } \\
& \int_{0}^{\mathrm{x}} \overline{\mathrm{F}}(\mathrm{u}) \mathrm{du} \leq \int_{0}^{\mathrm{x}} \overline{\mathrm{G}}(\mathrm{u}) \mathrm{du} .
\end{aligned}
$$


Another important ordering that has come to use in reliability and life testing is the following:

A random variable $\mathrm{X}$ is smaller than a random variable $\mathrm{Y}$ with respect to Laplace transform order (denoted by $\mathrm{X} \leq_{\mathrm{Lt}} \mathrm{Y}$ ) if, and only, if

$$
\int_{0}^{\infty} e^{-s x} d F(x) \geq \int_{0}^{\infty} e^{-s x} d G(x), \quad s \geq 0
$$

It is easy to check that (1.1) is equivalent to

$$
\int_{0}^{\infty} e^{-s x} \bar{F}(x) d x \leq \int_{0}^{\infty} e^{-s x} \bar{G}(x) d x
$$

Two classes of life distributions were introduced by Alzaid (1994) which are used better than aged (UBA) and used better than aged in expectation (UBAE) classes of life distribution.

Precisely we have the following definitions:

Definition (1.1): The df $\mathrm{F}$ is said to be used better than aged (UBA) if $0<\mu(\infty)<\infty$ and for all $\mathrm{x}, \mathrm{t} \geq 0$, (See Ahmad (2004))

$$
\overline{\mathrm{F}}(\mathrm{x}+\mathrm{t}) \geq \overline{\mathrm{F}}(\mathrm{t}) \mathrm{e}^{-\mathrm{x} / \mu(\infty)}, \mathrm{x}, \mathrm{t} \geq 0
$$

Definition (1.2): The distribution function $F$ is said to be used better than aged in expectation (UBAE) if $0<\mu(\infty)<$ $\infty$ and for all $\mathrm{x}, \mathrm{t} \geq 0$

$$
\mu(\mathrm{t}) \geq \mu(\infty)
$$

Note that, $F$ is UBA (UBAE) if and only if $X_{t}$ converges in distribution to a random variable $X_{A}$ (say) exponentially distributed with failure rate $1 / \mu$ and

$$
X_{t} \leq_{s t} X_{A},\left(E\left(X_{t}\right) \leq_{s t} E\left(X_{A}\right)\right)
$$

According to the above definitions (1.1), (1.2) and (1.3) we can deduce the following new definition for used better than aged in the Laplace transform order as follows.

Definition (1.3): The distribution function $\mathrm{F}$ is said to be used better than aged in the Laplace transform order (UBAL) if $0<\mu(\infty)<\infty$ and for all $\mathrm{x}, \mathrm{t} \geq 0$,

$\int_{0}^{\infty} \mathrm{e}^{-\mathrm{sx}} \overline{\mathrm{F}}(\mathrm{x}+\mathrm{t}) \mathrm{dx} \geq \frac{\mu(\infty)}{1+\mathrm{s} \mu(\infty)} \overline{\mathrm{F}}(\mathrm{t}), \mathrm{s} \geq 0$

It is obvious that (1.3) is equivalent to $\boldsymbol{X}_{\boldsymbol{t}} \leq_{\boldsymbol{L} t} \boldsymbol{X}_{\boldsymbol{A}}$ for all $\mathrm{t} \geq$ 0 .

To introduce the definition of the discrete UBAL, let $\mathrm{X}$ be a discrete non-negative random variable such that

$\mathrm{P}(\mathrm{X}=\mathrm{k})=\mathrm{p}_{\mathrm{k}}, \mathrm{k}=0,1,2, \ldots$. Let

$\overline{\mathrm{P}}_{\boldsymbol{k}}=\boldsymbol{P}(\boldsymbol{X}>k), \boldsymbol{k} \geq \mathbf{1}, \overline{\mathrm{P}}_{0}=1$ denote the corresponding survival function.

The discrete non-negative random variable $X$ is said to be discrete used better than aged in Laplace transform order (discrete UBAL) if, and only, if

$\sum_{\boldsymbol{k}=\mathbf{0}}^{\infty} \overline{\mathrm{P}}_{k+i} z^{k} \geq \overline{\mathrm{P}}_{i} \sum_{\boldsymbol{k}=\mathbf{0}}^{\infty} z^{k}$, for all $\mathbf{0} \leq \boldsymbol{z} \leq \mathbf{1}$ and $\boldsymbol{i}=$ $\mathbf{0}, \mathbf{1}, \ldots$.

Now,

$X \leq_{s t} X_{A} \Rightarrow X \leq_{L t} X_{A}$

Then, we have the following implication: (Abu-Youssef and Bakr $(2014,2018)$ and Abu-Youssef et al $(2015,2017))$.
IFR $\subset \quad$ UBA $\subset$ UBAL

UBAE

Applications, properties and interpretations of the Laplace transform order in the statistical theory of reliability, and in economics can be found in Denuit (2001), Klefsjo (1998) and Ahmed and Kayid (2004).

The main object in this paper is to deal with the problem of testing $\mathrm{H}_{0}: \mathrm{F}$ is exponential against $\mathrm{H}_{1}: \mathrm{F}$ is the largest class of life distribution UBAL. The paper is organized as follows: in section 2, we give a test statistic based on Laplace Transform technique for complete data. Selected critical values are tabulated for sample sizes 5(5)100 is investigated using mathematica 8 programme in section 3. The Pitman asymptotic efficiency for common alternatives is obtained in section 4. In section 5; a proposed test is presented for right censored data. Finally, we discuss some applications (numerical examples) to show the importance of the proposed test in section 6 .

\section{TESTING FOR COMPLETE DATA}

This section is concerned with the construction of the proposed statistic as a Laplace Transform technique and discussing its asymptotic normality.

Here, we hope to test the null hypothesis $H_{0}: F$ is exponential, against $H_{1}: F$ is UBAL, and is not exponential. Non-parametric testing for classes of life distributions has been considered by many authors (see Mahmoud and Abdul Alim (2008); Abu-Youssef and Bakr (2014, 2018); Abu-Youssef et al (2015)).

According to Eq. (1.5) we may use the following as a measure of departure from $H_{0}$.

$$
\begin{gathered}
\delta_{L}(\mathrm{~s}, \beta)=\int_{0}^{\infty}\left[\int_{0}^{\infty} e^{-\beta t} e^{-s x} \overline{\mathrm{F}}(x+t) \mathrm{d} x\right. \\
\left.-\frac{\mu(\infty)}{1+s \mu(\infty)} e^{-\beta t} \overline{\mathrm{F}}(t)\right] \mathrm{d} t \\
=\int_{0}^{\infty} \int_{0}^{\infty} e^{-\beta t} e^{-s x} \overline{\mathrm{F}}(x+t) d x d t-\frac{\mu(\infty)}{1+s \mu(\infty)} \int_{0}^{\infty} \mathrm{e}^{-\beta \mathrm{t}} \overline{\mathrm{F}}(\mathrm{t}) d t .
\end{gathered}
$$

The following theorem is essential for the development of our test statistic.

Theorem 4: Let $X$ be the UBAL random variable with distribution function $\mathrm{F}$; then based on the previous technique,

$$
\begin{aligned}
\delta_{L}(\mathrm{~s}, \beta)=\frac{1}{s(\beta-\mathrm{s})} & (1-\varphi(\mathrm{s})) \\
& -\left(\frac{1+\beta+s(1-\mu(\infty))}{\beta(\beta-s)(1+s \mu(\infty))}\right)(1-\varphi(\beta))
\end{aligned}
$$

Where $\varphi(s)=\int_{0}^{\infty} e^{-s x} d F(x)$.

Proof: Since 


$$
\begin{gathered}
\delta_{L}(\mathrm{~s}, \beta)=\int_{0}^{\infty} \int_{0}^{\infty} e^{-\beta t} \mathrm{e}^{-\mathrm{su}} \overline{\mathrm{F}}(\mathrm{u}+\mathrm{t}) \mathrm{dudt} \\
\quad-\frac{\mu(\infty)}{1+s \mu(\infty)} \int_{0}^{\infty} e^{-\beta t} \overline{\mathrm{F}}(t) \mathrm{d} t . \\
=I-\frac{\mu(\infty)}{1+s \mu(\infty)} I I .
\end{gathered}
$$

Where,

$$
\begin{aligned}
I & =\int_{0}^{\infty} \int_{0}^{\infty} e^{-\beta t} \mathrm{e}^{-\mathrm{su}} \overline{\mathrm{F}}(\mathrm{u}+\mathrm{t}) \mathrm{dudt} \\
& =\int_{0}^{\infty} \int_{\mathrm{t}}^{\infty} e^{-\beta t} \mathrm{e}^{-\mathrm{s}(\mathrm{x}-\mathrm{t})} \overline{\mathrm{F}}(\mathrm{x}) \mathrm{dxdt} \\
& =\frac{1}{\beta-s} \int_{0}^{\infty} e^{-s t}\left(1-\mathrm{e}^{-(\beta-\mathrm{s}) \mathrm{t}}\right) \overline{\mathrm{F}}(\mathrm{t}) \mathrm{dt} \\
& =\frac{1}{\beta-s}\left(\frac{1}{\mathrm{~s}}(1-\varphi(\mathrm{s}))-\frac{1}{\beta}(1-\varphi(\beta))\right) .
\end{aligned}
$$

And

$$
\begin{aligned}
I I & =\int_{0}^{\infty} e^{-\beta t} \overline{\mathrm{F}}(t) \mathrm{d} t \\
& =\frac{1}{\beta}(1-\varphi(\beta)) .
\end{aligned}
$$

Hence, the result follows.

Let $X_{1}, X_{2}, \ldots, X_{n}$ be a random sample from the distribution function $\mathrm{F}$.

For generality, we assume $\mu(\infty)$ is known and equal one. The empirical estimator

$\widehat{\delta}(\mathrm{s}, \beta)$ of our test statistic can be obtained as follows:

$$
\begin{aligned}
\widehat{\delta}_{L_{n}}(\mathrm{~s}, \beta)=\frac{1}{n(\beta-s)} \sum_{\mathrm{i}}\left\{\frac{1}{s}\left(1-\mathrm{e}^{-\mathrm{sX} \mathrm{X}_{\mathrm{i}}}\right)\right. \\
\left.-\frac{\beta+1}{\beta(1+\mathrm{s})}\left(1-\mathrm{e}^{-\beta \mathrm{X}_{\mathrm{i}}}\right)\right\} .
\end{aligned}
$$

To make the test is invariant, let

$$
\widehat{\Delta}_{L_{n}}(\mathrm{~s}, \beta)=\frac{\widehat{\delta}_{L_{n}}(\mathrm{~s}, \beta)}{\overline{\mathrm{X}}}
$$

Let us rewrite $\widehat{\delta}_{L}$ as follows,

$$
\widehat{\Delta}_{L_{n}}(\mathrm{~s}, \beta)=\frac{1}{\overline{\mathrm{X}} n} \sum_{\mathrm{i}} \emptyset\left(\mathrm{X}_{\mathrm{i}}\right)
$$

where

$$
\varnothing\left(\mathrm{X}_{\mathrm{i}}\right)=\frac{1}{\beta-s}\left\{\frac{1}{s}\left(1-\mathrm{e}^{-\mathrm{sX} \mathrm{X}_{\mathrm{i}}}\right)-\frac{\beta+1}{\beta(1+\mathrm{s})}\left(1-\mathrm{e}^{-\beta \mathrm{X}_{\mathrm{i}}}\right)\right\} .
$$

To find the limiting distribution of $\widehat{\delta}(\mathrm{s}, \beta)$ we resort to the Ustatistic theory and practice (Lee (1990)).

Set

$$
\varnothing\left(\mathrm{X}_{1}\right)=\frac{1}{\beta-s}\left\{\frac{1}{s}\left(1-\mathrm{e}^{-\mathrm{sX}_{1}}\right)-\frac{\beta+1}{\beta(1+\mathrm{s})}\left(1-\mathrm{e}^{-\beta \mathrm{X}_{1}}\right)\right\} .
$$

Then, $\widehat{\Delta}_{L_{n}}(\mathrm{~s}, \beta)$ is equivalent to U-statistic given by:

$$
U_{n}=\frac{1}{\left(\begin{array}{l}
n \\
1
\end{array}\right)} \sum_{\mathrm{i}} \emptyset\left(\mathrm{X}_{\mathrm{i}}\right) .
$$

The following theorem summarizes the asymptotic normality of $\widehat{\delta}_{L_{n}}(\mathrm{~s}, \beta)$.

\section{Theorem 5.}

i- As $\mathrm{n} \rightarrow \infty$, $\left(\widehat{\delta}_{L_{n}}(\mathrm{~s}, \beta)-\delta_{L}(\mathrm{~s}, \beta)\right)$ is asymptotically normal with mean 0 and variance $\sigma^{2}(\mathrm{~s}, \beta)$ where, $\sigma^{2}(\mathrm{~s}, \beta)=\operatorname{Var}\left[\hat{\delta}_{n}(\mathrm{~s}, \beta)\right]=$

ii- Under $H_{0}$, the variance is given by

$$
\begin{aligned}
& =\mathrm{E}\left(\frac { 1 } { \beta - s } \left\{\frac{1}{s}\left(1-\mathrm{e}^{-s \mathrm{X}_{\mathrm{i}}}\right)\right.\right. \\
& \left.\left.-\frac{\beta+1}{\beta(1+\mathrm{s})}\left(1-\mathrm{e}^{-\beta \mathrm{X}_{\mathrm{i}}}\right)\right\}\right)^{2}
\end{aligned}
$$

$$
\sigma_{0}^{2}(s, \beta)=\frac{2}{(2 \mathrm{~b}+1)(2 s+1)(s+1)^{2}(s+b+1)} .
$$

Proof:

i- Using standard U-statistic theory, Lee (1990) and direct calculations, we get

$$
\begin{array}{r}
E\left[\widehat{\delta}_{n}(\mathrm{~s}, \beta)\right]=E\left(\frac { 1 } { \beta - s } \left\{\frac{1}{s}\left(1-\mathrm{e}^{-s \mathrm{X}_{\mathrm{i}}}\right)\right.\right. \\
\left.\left.-\frac{\beta+1}{\beta(1+\mathrm{s})}\left(1-\mathrm{e}^{-\beta \mathrm{X}_{\mathrm{i}}}\right)\right\}\right) ; \\
\sigma^{2}(\mathrm{~s}, \beta)=\operatorname{Var}\left[\widehat{\delta}_{n}(\mathrm{~s}, \beta)\right]= \\
=\mathrm{E}\left(\frac { 1 } { \beta - s } \left\{\frac{1}{s}\left(1-\mathrm{e}^{-\mathrm{sX} \mathrm{X}_{\mathrm{i}}}\right)\right.\right. \\
\left.\left.-\frac{\beta+1}{\beta(1+\mathrm{s})}\left(1-\mathrm{e}^{-\beta \mathrm{X}_{\mathrm{i}}}\right)\right\}\right)^{2} .
\end{array}
$$

ii- $\quad$ Under $H_{0}$, the parameter $s=0.6$, $\beta=0.1$ say, and

$$
\begin{gathered}
\mu_{0}=E\left[\widehat{\delta}_{n}(\mathrm{~s})\right]=0 ; \\
\sigma_{0}^{2}(0.6,0.1)=\frac{2}{(2 \mathrm{~b}+1)(2 s+1)(s+1)^{2}(s+b+1)} \\
=0.17 .
\end{gathered}
$$

\section{MONTE CARLO NULL}

\section{DISTRIBUTION CRITICAL POINTS}

Based on 10000 generated samples from the standard exponential distribution the Monte Carlo null distribution critical values of our test $\widehat{\delta}_{L_{n}}(0.6,0.1)$ are simulated and tabulated, where $\mathrm{n}=5(5) 100$ in Table 1 . Mathematica 8 programme is used.

\begin{tabular}{|l|l|l|l|}
\hline $\mathrm{n}$ & $90 \%$ & $95 \%$ & $99 \%$ \\
\hline 5 & 0.178107 & 0.22842 & 0.307209 \\
\hline 10 & 0.129312 & 0.166508 & 0.227719 \\
\hline 15 & 0.121644 & 0.151435 & 0.202949 \\
\hline 20 & 0.0973733 & 0.132505 & 0.191256 \\
\hline 25 & 0.0835908 & 0.107235 & 0.145443 \\
\hline 30 & 0.0870262 & 0.112839 & 0.171846 \\
\hline 35 & 0.0806331 & 0.106212 & 0.145184 \\
\hline 40 & 0.0767111 & 0.137902 & 0.137902 \\
\hline
\end{tabular}




\begin{tabular}{|l|l|l|l|}
\hline 45 & 0.0690628 & 0.0903939 & 0.124415 \\
\hline 50 & 0.067212 & 0.0888034 & 0.127334 \\
\hline 55 & 0.0649281 & 0.0839744 & 0.117099 \\
\hline 60 & 0.0627974 & 0.0787144 & 0.111644 \\
\hline 65 & 0.058578 & 0.0778206 & 0.0998889 \\
\hline 70 & 0.062877 & 0.0755414 & 0.113515 \\
\hline 75 & 0.0522209 & 0.0693114 & 0.101782 \\
\hline 80 & 0.0537328 & 0.0711821 & 0.101084 \\
\hline 85 & 0.0522313 & 0.066287 & 0.0913245 \\
\hline 90 & 0.0548479 & 0.0691084 & 0.0937278 \\
\hline 95 & 0.051652 & 0.0672995 & 0.0880698 \\
\hline 100 & 0.046207 & 0.0604187 & 0.0856818 \\
\hline
\end{tabular}

Table 1: The Upper Percentile Points of $\widehat{\delta}_{L_{n}}(0.6,0.1)$ with 10000 Replications.

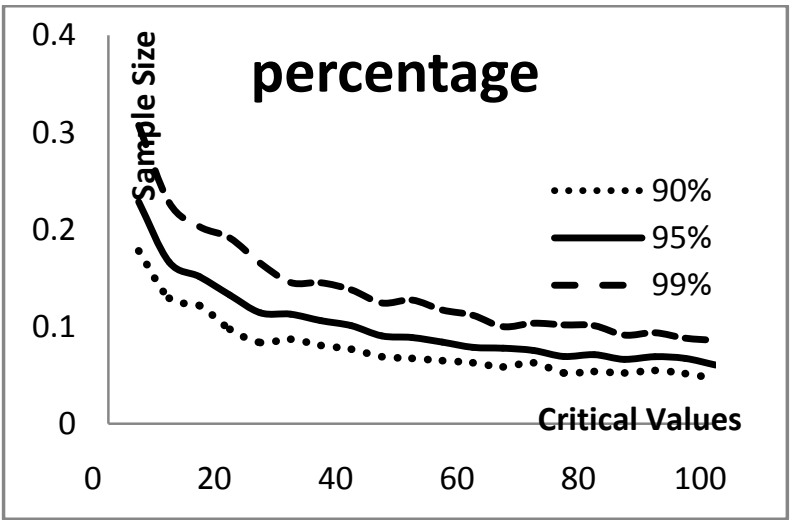

Fig. 1: The Relation Between Sample Size and Critical Values.

From Table 1 and Fig. 1, the critical values decrease as the sample size increases and they increase as the confidence level increases.

\section{PITTMAN ASYMPTOTIC RELATIVE EFFICIENCY}

Since the above test statistic $\widehat{\Delta}_{L}(\mathrm{~s}, \beta)=\frac{\widehat{\delta}_{L}}{\overline{\mathrm{X}}}$ is new and no other tests are known for this class (UBAL). We may compare our test to the other classes. Here we choose the test $\Delta_{\theta,(1)}$ presented by Mugdadi and Ahmad (2005) and $\delta_{\mathrm{F}_{\mathrm{n}}}^{(2)}$ presented Mahmoud and Abdul Alim (2008) for (NBAFR) class of life distribution. Then comparisons are achieved by using Pitman asymptotic relative efficiency PARE, which is defined as follows:

Let $\mathrm{T}_{1 \mathrm{n}}$ and $\mathrm{T}_{2 \mathrm{n}}$ be two statistics, then PARE of $\mathrm{T}_{1 \mathrm{n}}$ relative to $\mathrm{T}_{2 \mathrm{n}}$ is defined by

$$
\mathrm{e}\left(\mathrm{T}_{1 \mathrm{n}}, \mathrm{T}_{2 \mathrm{n}}\right)=\frac{\mu_{1}^{\backslash}\left(\theta_{0}\right)}{\sigma_{1}\left(\theta_{0}\right)} / \frac{\mu_{2}^{\backslash}\left(\theta_{0}\right)}{\sigma_{2}\left(\theta_{0}\right)} .
$$

Where $\mu_{\mathrm{i}} \backslash\left(\theta_{0}\right)=\left.\lim _{\mathrm{n} \rightarrow \infty} \frac{\partial}{\partial \theta} \mathrm{E}\left(\mathrm{T}_{\mathrm{n}_{\mathrm{i}}}\right)\right|_{\theta \rightarrow \theta_{0}}$, and

$$
\sigma_{\mathrm{i}}^{2}\left(\theta_{0}\right)=\lim _{\mathrm{n} \rightarrow \infty} \operatorname{var}\left(\mathrm{T}_{\mathrm{n}_{\mathrm{i}}}\right) .
$$

Three of the most commonly used alternatives they are:

(i) Linear failure rate family

$$
\overline{\mathrm{F}}_{1}(\mathrm{x})=\mathrm{e}^{-\mathrm{x}-\frac{\mathrm{x}^{2}}{2} \theta}, \quad \theta, \mathrm{x} \geq 0 .
$$

(ii) Weibull family:

$$
\overline{\mathrm{F}}_{2}(\mathrm{x})=\mathrm{e}^{-\mathrm{x}^{\theta}}, \quad \theta \geq 1,, \mathrm{x} \geq 0 .
$$

(iii) Makeham family:

$$
\overline{\mathrm{F}}_{2}(\mathrm{x})=\mathrm{e}^{-\mathrm{x}-\theta\left(\mathrm{x}+\mathrm{e}^{-\mathrm{x}}-1\right)}, \quad \theta, \mathrm{x} \geq 0 .
$$

Note that $\mathrm{H}_{0}$ (the exponential distribution) is attained at $\theta=0$ in (i), (iii) and $\theta=1$ in (ii). The Pitman's asymptotic efficiency (PAE) of $\delta_{L}(\mathrm{~s}, \beta)$ is equal to

$$
\begin{gathered}
\operatorname{PAE}\left(\delta_{L}(\mathrm{~s}, \beta)\right)=\frac{\left|\frac{\partial}{\partial \theta} \delta_{L}(\mathrm{~s}, \beta)\right|_{\theta \rightarrow \theta_{0}}}{\sigma_{0}(\mathrm{~s}, \beta)}= \\
=\frac{1}{\sigma_{0}(\mathrm{~s}, \beta)} \mid \frac{1}{\mathrm{~s}(\beta-\mathrm{s})} \int_{0}^{\infty} \mathrm{e}^{-\mathrm{sx}} \mathrm{d} \overline{\mathrm{F}}_{\theta_{0}}(\mathrm{x}) \\
-\frac{\beta+1}{\beta(1+s)(\beta-s)} \int_{0}^{\infty} \mathrm{e}^{-\beta \mathrm{x}} \mathrm{d} \overline{\mathrm{F}}_{\theta_{0}}(\mathrm{x}) \mid
\end{gathered}
$$

Where $\overline{\mathrm{F}}_{\theta_{0}}(\mathrm{x})=\left.\frac{\mathrm{d}}{\mathrm{d} \theta} \overline{\mathrm{F}}_{\theta}(\mathrm{u})\right|_{\theta \rightarrow \theta_{0}}$

This leads to:

(i) PAE in case of the linear failure rate distribution:

$$
\begin{aligned}
\operatorname{PAE}\left(\delta_{L}(0.6,0.1)\right) & =\frac{1}{\sigma_{0}(0.6,0.1)} \mid \frac{-1}{0.3} \int_{0}^{\infty} \mathrm{e}^{-0.6 \mathrm{x}} \mathrm{d}\left(-\frac{\mathrm{x}^{2}}{2} \mathrm{e}^{-\mathrm{x}}\right) \\
& -\frac{-1.1}{0.08} \int_{0}^{\infty} \mathrm{e}^{-0.1 \mathrm{x}} \mathrm{d}\left(-\frac{\mathrm{x}^{2}}{2} \mathrm{e}^{-\mathrm{x}}\right) \mid=1.77
\end{aligned}
$$

(ii) PAE in case of the Weibull distibution:

$$
\begin{aligned}
& \operatorname{PAE}\left(\delta_{L}(0.6,0.1)\right)= \\
& =\frac{1}{\sigma_{0}(0.6,0.1)} \mid \frac{-1}{0.3} \int_{0}^{\infty} \mathrm{e}^{-0.6 \mathrm{x}} \mathrm{d}\left(-\mathrm{x} \ln (\mathrm{x}) \mathrm{e}^{-\mathrm{x}}\right) \\
& -\frac{-1.1}{0.08} \int_{0}^{\infty} \mathrm{e}^{-0.1 \mathrm{x}} \mathrm{d}\left(-\mathrm{x} \ln (\mathrm{x}) \mathrm{e}^{-\mathrm{x}}\right) \mid=0.98
\end{aligned}
$$

(iii) PAE in case of the Makeham distribution.

$$
\begin{aligned}
\operatorname{PAE}(\delta(0.6,0.1))= & \frac{1}{\sigma_{0}} \mid \frac{-1}{0.3} \int_{0}^{\infty} \mathrm{e}^{-0.6 \mathrm{x}} \mathrm{d}\left(\left(1-\mathrm{x}-\mathrm{e}^{-\mathrm{x}}\right) \mathrm{e}^{-\mathrm{x}}\right) \\
& -\frac{-1.1}{0.08} \int_{0}^{\infty} \mathrm{e}^{-0.1 \mathrm{x}} \mathrm{d}\left(\left(1-\mathrm{x}-\mathrm{e}^{-\mathrm{x}}\right) \mathrm{e}^{-\mathrm{x}}\right) \mid \\
& =0.58
\end{aligned}
$$

Direct calculations of PAE of $\Delta_{\theta,(1)}, \delta_{\mathrm{F}_{\mathrm{n}}}^{(2)}$ and $\delta_{L}(\mathrm{~s}, \beta)$ are summarized in table (2), the efficiencies in table shows clearly our test $\delta_{L}(\mathrm{~s}, \beta)$ perform well for $\mathrm{F}_{1}, \mathrm{~F}_{2}$ and $\mathrm{F}_{3}$. 


\begin{tabular}{l|lll|}
\hline Distribution & \multicolumn{1}{|c|}{$\Delta_{\theta,(1)}$} & $\delta_{\mathrm{F}_{\mathrm{n}}}^{(2)}$ & $\delta_{L}(\mathrm{~s}, \boldsymbol{\beta})$ \\
\hline LFR & 0.408 & 0.217 & 1.77 \\
Weibull & 0.170 & 0.050 & 0.98 \\
Makeham & 0.0395 & 0.144 & 0.58 \\
\hline
\end{tabular}
Table 2: PAE of $\Delta_{\boldsymbol{\theta},(\mathbf{1})}, \boldsymbol{\delta}_{\mathbf{F}_{\mathbf{n}}}^{(2)}$ and $\boldsymbol{\delta}_{\boldsymbol{L}}(\mathbf{s}, \boldsymbol{\beta})$

In table (3), we give PARE's of $\delta_{L}(\mathrm{~s}, \beta)$ with respect to $\Delta_{\theta,(1)}$ and $\delta_{\mathrm{F}_{\mathrm{n}}}^{(2)}$ whose PAE are mentioned in table 2 .

\begin{tabular}{|l|ll|}
\hline Distribution & $\mathrm{e}\left(\delta_{L}(\mathrm{~s}, \beta), \Delta_{\theta,(1)}\right)$ & $\mathrm{e}\left(\delta_{L}(\mathrm{~s}, \beta), \delta_{\mathrm{F}_{\mathrm{n}}}^{(2)}\right)$ \\
\hline LFR & 4.3 & 7.8 \\
Weibull & 5.76 & 19.6 \\
Makeham & 14.68 & 4.03 \\
\hline
\end{tabular}

Table 3: PARE of $\delta_{L}(s, \beta)$ with respect to $\Delta_{\theta,(1)}$ and $\delta_{F_{n}}^{(2)}$.

It is clear from table (3) that the statistic $\delta_{L}(s, \beta)$ perform well for $\bar{F}_{1}, \bar{F}_{2}$ and $\bar{F}_{3}$ and it is more efficient than both $\Delta_{\theta,(1)}$ and $\delta_{\mathrm{F}_{\mathrm{n}}}^{(2)}$ for all cases mentioned above. Hence our test, which deals the much larger UBA is better and also simpler.

\section{TESTING FOR RIGHT CENSORED \\ DATA}

In this section, a test statistic is proposed to test: $\mathrm{H}_{0}(\overline{\mathrm{F}}$ is exponential distribution with mean $\mu)$ versus $H_{1}(\bar{F}$ is UBAL and not exponential distribution); with randomly rightcensored data.

It is known that a censored data is usually the only information available in a life testing model or in a clinical study where patients may be lost (censored) before the completion of a study. We can describe the experimental situation as follows. Suppose $\mathrm{n}$ units are put on test, and $\mathrm{X}_{1}, \mathrm{X}_{2}, \ldots, \mathrm{X}_{\mathrm{n}}$ denote their true life time. Let that $\mathrm{X}_{1}, \mathrm{X}_{2}, \ldots, \mathrm{X}_{\mathrm{n}}$ be independent and identically distributed (i.i.d.) according to a continuous life distribution $\mathrm{F}$.

Let $Y_{1}, Y_{2}, \ldots, Y_{n}$ be (i.i.d.) according to a continuous life distribution $\mathrm{G}$. Also we assume

that $\mathrm{X}, \mathrm{s}$ and $\mathrm{Y}, \mathrm{s}$ are independent. In the randomly right censored model, we observe the pairs

$\left(\mathrm{Z}_{\mathrm{i}}, \delta_{\mathrm{i}}\right), i=1, \ldots, n$ where $\mathrm{Z}_{\mathrm{i}}=\min \left(\mathrm{X}_{\mathrm{i}}, \mathrm{Y}_{\mathrm{i}}\right)$ and we assume $\mu(\infty)$ is known and equal one. and

$$
\delta_{\mathrm{i}}=\left\{\begin{array}{l}
1 \quad \text { if } \quad \mathrm{Z}_{\mathrm{i}}=\mathrm{X}_{\mathrm{i}} \text { (i th observation is uncensored) } \\
0 \quad \text { if } \quad \mathrm{Z}_{\mathrm{i}}=\mathrm{Y}_{\mathrm{i}} \text { (i th observation is censored). }
\end{array}\right.
$$

Let $\mathrm{Z}_{(0)}<\mathrm{Z}_{(1)}<\ldots<\mathrm{Z}_{(\mathrm{n})}$ denoted the ordered of Z's and $\delta_{\mathrm{i}}$ is the $\delta$ corresponding to $\mathrm{Z}_{(\mathrm{i})}$, respectively. Using the Kaplan and Meier estimator in the case of censored data $\left(\mathrm{Z}_{\mathrm{i}}, \delta_{\mathrm{i}}\right), \mathrm{i}=1, \ldots, \mathrm{n}$ as follows:

$$
\begin{aligned}
& \delta_{\mathrm{c}}(s)=\frac{1}{(\beta-s)} \sum_{j=1}^{l} \prod_{k=1}^{j-1}\left(\frac{1}{s}(1\right. \\
& \left.-\sum_{m=1}^{l} e^{-s Z_{(m)}}\left[\prod_{p=1}^{m-2} C_{p}^{\delta_{p}}-\prod_{p=1}^{m-1} C_{p}^{\delta_{p}}\right]\right) \\
& -\frac{\beta+1}{\beta(1+s)}(1 \\
& \left.\left.-\sum_{m=1}^{l} e^{-\beta Z_{(m)}}\left[\prod_{p=1}^{m-2} C_{p}^{\delta_{p}}-\prod_{p=1}^{m-1} C_{p}^{\delta_{p}}\right]\right)\right) .
\end{aligned}
$$

Where

$$
\begin{gathered}
\varphi(\mathrm{s})=\int_{0}^{\infty} \mathrm{e}^{-\mathrm{sx}} \mathrm{dF}(\mathrm{x}) \\
\widehat{\varphi}(\mathrm{s})=\sum_{m=1}^{l} e^{-s Z_{(m)}}\left(\prod_{p=1}^{m-2} C_{p}^{\delta_{p}}-\prod_{p=1}^{m-1} C_{p}^{\delta_{p}}\right) \\
\mathrm{d} F_{n}\left(Z_{i}\right)=\prod_{q=1}^{j-2} C_{i}^{\delta_{i}}-\prod_{q=1}^{j-1} C_{i}^{\delta_{i}}, \\
\bar{F}_{n}(t)=\prod_{m<t} C_{m}^{\delta_{m}}, \\
\frac{n-m}{n-m+1}, t \in\left[0, z_{(m)}\right] .
\end{gathered}
$$

\begin{tabular}{|l|l|l|l|}
\hline $\mathrm{n}$ & $95 \%$ & $98 \%$ & $99 \%$ \\
\hline 5 & 0.0486062 & 0.0639535 & 0.0778679 \\
\hline 10 & 0.0326447 & 0.0399905 & 0.0447338 \\
\hline 15 & 0.0312726 & 0.035816 & 0.0396453 \\
\hline 20 & 0.0287089 & 0.0315818 & 0.0342952 \\
\hline 25 & 0.0259153 & 0.027619 & 0.0293663 \\
\hline 30 & 0.0254728 & 0.0277263 & 0.0294407 \\
\hline 35 & 0.0250413 & 0.026873 & 0.0281711 \\
\hline 40 & 0.0240957 & 0.0262975 & 0.0273122 \\
\hline 45 & 0.0235821 & 0.0254789 & 0.0268156 \\
\hline 50 & 0.0234395 & 0.0247306 & 0.0258166 \\
\hline 55 & 0.0233212 & 0.0253982 & 0.026503 \\
\hline 60 & 0.0232044 & 0.0245652 & 0.0250577 \\
\hline 65 & 0.0225061 & 0.0240242 & 0.0249353 \\
\hline 70 & 0.0229756 & 0.0241366 & 0.0247014 \\
\hline 75 & 0.0223271 & 0.0233298 & 0.0239314 \\
\hline 80 & 0.0219044 & 0.0235257 & 0.0239446 \\
\hline 85 & 0.0221512 & 0.0229988 & 0.0235409 \\
\hline 90 & 0.0220748 & 0.0228619 & 0.0235677 \\
\hline
\end{tabular}




\begin{tabular}{|l|l|l|l|}
\hline 95 & 0.0216011 & 0.0225204 & 0.02321 \\
\hline 100 & 0.0215823 & 0.0224034 & 0.0231377 \\
\hline
\end{tabular}

Table 4: The Upper Percentile Points of $\widehat{\boldsymbol{\delta}}_{c}(10,100)$ with 10000 Replications

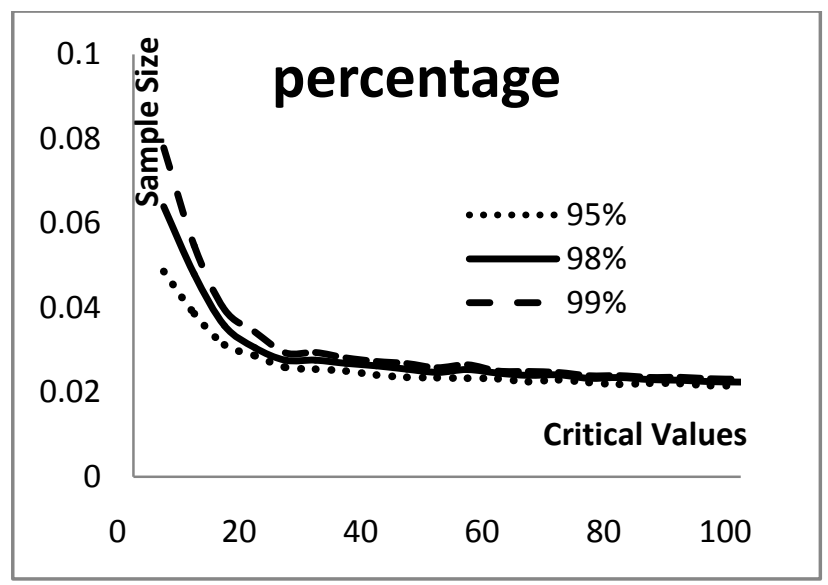

Fig. 2: The Relation Between Sample Size and Critical Values.

From Table 4 and Fig. 2, the critical values decrease as the sample size increases and they increase as the confidence level increases.

\section{APPLICATIONS}

Here, we introduce some of real examples to elucidate the applications of our test in the two cases (censored and noncensored data) of no censored data at $95 \%$ confidence level.

\section{a- Case of complete data}

In this section, two examples are presented considering

$\mathrm{s}=0.6, \mathrm{~b}=0.1$.

Example 1: Consider the data in Abouammoh et al. (1994) these data represent set of 40 patients suffering from blood cancer (leukemia) from one of ministry of health hospitals in Saudi Arabia and the ordered values in years are:

$\begin{array}{lllllll}0.315 & 0.496 & 0.616 & 1.145 & 1.208 & 1.263 & 1.414 \\ 2.025 & 2.036 & 2.162 & 2.211 & 2.370 & 2.532 & 2.693 \\ 2.805 & 2.910 & 2.912 & 3.192 & 3.263 & 3.348 & 3.348 \\ 3.427 & 3.499 & 3.534 & 3.767 & 3.751 & 3.858 & 3.986 \\ 4.049 & 4.244 & 4.323 & 4.381 & 4.392 & 4.397 & 4.647 \\ 4.753 & 4.929 & 4.973 & 5.074 & 4.381 & & \end{array}$

It was found that $\widehat{\delta}_{L}(0.6,0.1)=0.31$ which is greater than the critical value of Table 1 . Then we conclude that this data set have UBAL property and not exponential.

Example 2: Consider the well-known Darwin data Fisher (1966) that represent the differences in heights between cross and self-fertilized plants of the same pair grown together in one pot:

$\begin{array}{lllll}4.9 & -6.7 & 0.8 & 1.6 & 0.6 \\ 2.3 & 2.8 & 4.1 & 1.4 & 2.9 \\ 5.6 & 2.4 & 7.5 & 6.0 & -4.8\end{array}$

It was found that $\widehat{\delta}_{L}(0.6,0.1)=0.069$ which less than the critical value of Table 1 . Then, we accept the null hypotheses which states that the data set has exponential property.

\section{b- Case of censored data}

In this section, an example is presented considering $\mathrm{s}=10$, $\mathrm{b}=100$.

Example 3: Consider the data in Mahmoud and Abdul Alim (2008) which represent 51 liver cancers patients taken from Elminia cancer center Ministry of Health \{ Egypt, which entered in (1999). Of them 39 represent whole life times (noncensored data) and the others represent censored data. The ordered life times (in days) are The ordered non-censored data are

$\begin{array}{lllllllll}10 & 14 & 14 & 14 & 14 & 14 & 15 & 17 & 18 \\ 20 & 20 & 20 & 20 & 20 & 23 & 23 & 24 & 26 \\ 30 & 30 & 31 & 40 & 49 & 51 & 52 & 60 & 61 \\ 67 & 71 & 74 & 75 & 87 & 96 & 105 & 107 & 107 \\ 107 & 116 & 150 & & & & & & \end{array}$

The ordered censored data are:

$\begin{array}{llllll}30 & 30 & 30 & 30 & 30 & 60 \\ 150 & 150 & 150 & 150 & 150 & 185\end{array}$

One can calculate $\widehat{\delta}_{c}(10,100)=0.11$ which is greater than the critical value of Table 4 . Then we conclude that this data set have UBAL property and not exponential.

\section{ACKNOWLEDGEMENTS}

The authors would like to thank the editors and the referees for their valuable comments and suggestions.

\section{REFERENCES}

[1] Abouammoh AM., Abdulghani SA., Qamber IS. (1994). On partial orderings and testing of new better than renewal used classes. Reliability Eng. Syst. Safety 43:3741.

[2] Abu-Youssef, S. E. and Bakr, M.E. (2014). Some properties of UBACT class of life distribution. $J$. of Adva. Res. in Appl. Math. 1-9.

[3] Abu-Youssef, S. E. and Bakr, M.E. (2014). A goodness of fit approach to class of life distribution with unknown age. Int. J. of Comp. App., 30-35.

[4] Abu-Youssef, S. E., Mohammed, B. I. and Bakr, M.E. (2014). Nonparametric test for unknown age class of life distributions. Int. J. of Rel. and Appl., 99-110.

[5] Abu-Youssef, S. E., Mohammed, B. I. and Bakr, M.E. (2015). Nonparametric Test for UBACT Class of Life Distribution Based on U-Statistic. J. of adv. of math., 402-411.

[6] Abu-Youssef, S. E. and Bakr, M.E. (2018). Laplace Transform Order for Unknown Age Class of Life Distribution with Some Applications. J. Stat. Appl. Pro. 1-10.

[7] Abu-Youssef, S. E., Nahed, S. A. and Bakr, M.E. (2017). Non Parametric Testing for Unknown age class of life distribution. Submitted.

[8] Abu-Youssef, S. E., Nahed, S. A. and Bakr, M.E. (2017). Used Better than Aged in mgf Ordering Class of Life Distribution with Application of Hypothesis Testing. Submitted. 
[9] Ahmad, I. A. (2004). Some properties of classes of life distributions with unknown age. J. Statist. Prob., 333342 .

[10] Ahmed H. and Kayid M. (2004). Preservation properties for the Laplace transform ordering of residual lives. Statist. Pap. 583-590.

[11] Alzaid, A. A. (1994). Aging concepts for item of unknown age. Stochastic models, 649-659.

[12] Barlow, R. E. and Proschan, F. (1981). Statistical theory of reliability and life testing: Probability models, To Begin With, Silver Spring, MD.

[13] Bryson, M. C. and Siddiqui, M. M. (1969). Some criteria for aging. J. of Ame. Sta. Ass. 1472-1483.

[14] Denuit M. (2001). Laplace transform ordering of actuarial quantities. Insur Math Econ. 83-102.

[15] Fisher RA. (1966). The design of experiments eight edition. Oliver \& Boyd, Edinburgh.
[16] Klefsjo, B. (1983). A useful aging property based on the Laplace transform. J. Appl. Prob. 615-626.

[17] Lee (1990). U-statistics theory and practice. Marcel Dekker, New York.

[18] Mahmoud, M. A. W. and Abdul Alim, A. N. (2008). A Goodness of Fit Approach to for Testing NBUFR (NWUFR) and NBAFR (NWAFR) Properties, Int. $J$. Reliab. Appl. 125-140.

[19] Mugdadi, A. R. and Ahmad, I. A. (2005). Moment Inequalities Derived from Comparing Life with Its Equlibrium Form. J. Stat. Plann. Inf. 303-317.

[20] Willmot, G.E. and Cai, J. (2000). On classes of lifetime distributions with unknown age. Probab. Eng. Inform. Sci. 473-484 